\title{
Excipiente para a produção de comprimidos por compressão direta
}

\author{
Excipients for direct compression tablet production
}

Tiago Rafael SAUSEN ${ }^{1 *}$, Paulo MAYORGA ${ }^{2}$

${ }^{I}$ Pontifícia Universidade Católica do Paraná - PUCPR, Av. da União, 500, 85902-

532, Toledo - PR, Brasil..E-mail: tiago.sausen@pucpr.br. ${ }^{2}$ Faculdade de Farmácia, Universidade Federal do Rio Grande do Sul-UFRGS

\section{ABSTRACT}

The pharmaceutical industry's interest in the tablet production by direct compression has increased in recent years, especially because it is an easy to control and less process time production method, leading to greater productivity of the production line. However, the successful production of tablets by direct compression relies almost exclusively of adjuvants that comprise the formulation. This article describes the production of tablets by direct compression, addressing the characteristics and key excipients involved in this process.

Keywords: excipients, pharmaceutical technology, tablets

\section{RESUMO}

O interesse da indústria farmacêutica na produção de comprimidos através da compressão direta tem aumentado nos últimos anos, principalmente por ser um método de produção de fácil controle e com menor tempo de processo, levando a uma maior produtividade da linha de produção. Entretanto, o sucesso da produção de comprimidos por compressão direta depende quase que exclusivamente dos adjuvantes que compõem a formulação. O presente artigo descreve a produção de comprimidos por compressão direta, abordando as características e os principais excipientes envolvidos nesse processo.

Palavras Chave: comprimidos, excipientes, tecnologia farmacêutica

\section{INTRODUÇÃO}

A compressão direta é um processo que envolve duas operações seqüenciais: mistura de pós e compressão (1). Quando comparada com o processo de granulação, a compressão direta oferece muitas vantagens, principalmente no que se refere às etapas de produção (Tabela 1). Em processos industriais, cada etapa adicional significa mais equipamentos, operadores, espaço, consumo de energia, validações de processos e, principalmente, custos, e cada passo pode diminuir o rendimento da produção e aumentar o surgimento de produtos fora de especificação (2). Na indústria farmacêutica, onde em alguns casos os produtos possuem baixas margens de lucro e o processo de produção é desenvolvido por bastan- te tempo, a utilização da compressão direta pode reduzir os custos, proporcionando um grande impacto nos lucros (3). Entretanto, a compressão direta não deve ser vista como uma simplificação do processo de granulação para a produção de comprimidos. O desenvolvimento de comprimidos por compressão direta necessita de uma avaliação crítica das matérias-primas envolvidas, das propriedades de fluxo das misturas de pós e dos efeitos das variáveis da formulação na compressibilidade (4).

Uma das vantagens mais significantes da compressão direta é que com a eliminação da etapa de granulação, aumenta a estabilidade de fármacos que podem degradar em decorrência do umedecimento e/ou da exposição ao calor (5). Dessa forma, a compressão direta é considerada a técnica de escolha para a produção de 
comprimidos contendo fármacos termolábeis e sensíveis à umidade (6). Outra vantagem da compressão direta é a otimização da desintegração dos comprimidos obtidos, pois eles se desintegram em partículas primárias ao invés de grânulos, gerando um aumento da área superficial para dissolução e pode resultar em uma liberação mais rápida do fármaco. Mudanças nos perfis de dissolução são menos prováveis de ocorrer em comprimidos fabricados por compressão direta do que os obtidos por processos de granulação $(7,8)$.

Tabela 1: Passos na produção de comprimidos por via granulação úmida e por compressão direta.

\begin{tabular}{l}
\multicolumn{1}{c}{ GRANULAÇÃo ÚMIDA } \\
\hline 1 - Mistura Inicial \\
2 - Umedecimento \\
3 - Granulação \\
4 - Secagem \\
5 - Tamisação \\
6 - Mistura Final (adição de lubrificantes) \\
7 - Compressão
\end{tabular}

A compressão direta também possui suas limitações, principalmente de ordem tecnológica. Comprimidos contendo uma alta dose de um fármaco com baixa compactabilidade, pobres propriedades de fluxo e/ou baixa densidade bruta não podem ser comprimidos diretamente (8). Para possibilitar um pó com boa capacidade de escoamento e densidade aceitáveis, partículas relativamente grandes podem ser usadas, as quais, primeiramente, podem ser propensas à segregação (5).

O fluxo de um pó e caracterizado como a sua capacidade de mover-se de um local para outro, seja durante o preparo da formulação (misturador, high-shear ou leito fluidizado), seja durante a produção da forma farmacêutica (encapsuladora ou compressora). As propriedades de fluxo de um pó podem ser determinadas através de diversos testes. Uma dessas técnicas é a determinação do ângulo de repouso, que depende, essencialmente, da força de fricção entre as partículas do pó, influenciando as características de fluxo e a estabilidade de empacotamento. Em linhas gerais, pode-se dizer que pós que apresentem ângulo de repouso com valor superior a $50^{\circ}$ possuem propriedades de fluxo insatisfatórias, enquanto pós com ângulo próximo a $25^{\circ}$ caracterizam-se por possuírem propriedades de fluxo muito boas (5).

Outra possibilidade de determinação é através dos cálculos do Fator de Hausner e do Índice de Carr $(9 ; 10)$. Para a obtenção desse cálculo utilizam-se os valores de densidade bruta e de densidade compactada. A diferença entre densidade bruta e densidade de compactação pode dar um indicativo sobre o comportamento do sistema de empacotamento e o fluxo de um material sólido particulado. Quanto maior for essa diferença, a teoria indica que melhor será o fluxo e menos estável será seu sistema de empacotamento $(11,12)$.

\section{COMPRESSÃO DIRETA}

1 - Mistura Inicial

2 - Mistura Final (adição de lubrificantes) 3 - Compressão

O fator de Hausner reflete condições de atrito entre as partículas do pó, o que depende do coeficiente de atrito do material, do tamanho e forma das partículas, assim como das propriedades de superfície das mesmas (13). É esperada uma maior estabilidade de empacotamento para sistemas com valores de $\mathrm{FH}$ mais próximos da unidade. Já o índice de Carr, que expressa a capacidade de densificação do pó, é uma avaliação indireta da estabilidade espacial do material, na medida em que reflete a tendência em formar aglomerados ou pontes que dificultariam a compressão (13). Valores de IC elevados (acima de $20 \%$ ) podem ser indicativos de produtos com baixa capacidade de escoamento enquanto valores baixos de IC indicam produtos com maior estabilidade de empacotamento (10).

Estes parâmetros estão intimamente ligados à dimensão, forma, condições de superfície e coeficiente de fricção das partículas. As condições do ensaio permitem estabelecer uma analogia com as etapas de enchimento em máquina de comprimir, relacionando os resultados diretamente à reprodutibilidade do processo (9). É importante ressaltar que estes parâmetros são meramente exploratórios. Tradicionalmente são empregados na caracterização tecnológica dos complexos farmacêuticos, não sendo capazes de fornecer informações mais precisas sobre os parâmetros representados.

A compressão direta pode apresentar alguns problemas devido às características do complexo farmacêutico pulverulento, tais como escassa capacidade de fluxo, possibilidade de aderência às ferramentas de compressão e baixa coesão, ligada à sua reduzida área superficial que diminui, consideravelmente, os pontos de contato no momento da compressão. Esses problemas podem ser contornados pela seleção da forma de partículas e de faixa granulométrica adequada das substâncias, através de dispositivos mecânicos (elevada força 
de compressão e emprego de acessórios que facilitem o enchimento das matrizes) e também, mais comumente utilizado, mediante o emprego de adjuvantes de maior eficiência $(14,15)$.

A obtenção de comprimidos requer que o material a comprimir possua propriedades físicas e mecânicas específicas, ou seja, capacidade de fluir livremente, coesividade e lubrificação. Como nem todos os fármacos possuem tais propriedades faz-se necessária a adição de adjuvantes farmacêuticos (16). Um dos mais importantes aspectos do desenvolvimento e produção de comprimidos é a escolha apropriada dos adjuvantes empregados na formulação. Propriedades físico-químicas como densidade, tamanho, forma, volume e distribuição das partículas, além das propriedades mecânicas, entre elas, compressibilidade e compactabilidade ou coesividade, ditam o comportamento da formulação durante a compressão e a forma como o sistema liberará o fármaco $(17,18)$.

Muitos fatores influenciam na escolha do excipiente a ser utilizado na fabricação de comprimidos, sendo que os requerimentos para adjuvantes de compressão direta incluem (5):

- Alta compactabilidade;

- Bom fluxo;

- Boas propriedades de mistura;

- Baixa sensibilidade a lubrificantes;

- Boa estabilidade;

- Ser inerte;

- Compatibilidade;

- Não interferência com a biodisponibilidade da substância ativa;

- Efeito promotor na desintegração do comprimido (se desejado);

- Efeito promotor na liberação do fármaco (se desejado);

- Disponibilidade comercial;

- Reprodutibilidade lote-a-lote;

- Custo efetivo.

\section{PRINCIPAIS EXCIPIENTES UTILIZADOS NA COMPRESSÃO DIRETA}

Os excipientes para compressão direta são desenvolvidos de forma que o pó misturado exiba boas propriedades de fluxo e de deformação e, por esse motivo, são usualmente mais caros do que os utilizados no processo de granulação úmida $(1,8)$. Esses excipientes não são apenas compressíveis sozinhos, mas também podem ser misturados a grandes proporções de um fármaco sem deterioração significativa na qualidade do comprimido $(6,19)$.

Quando se pretende produzir comprimidos por compressão direta, a escolha do material de carga ou en- chimento é extremamente crítica, pois, normalmente, é o componente presente na formulação em maior quantidade. Ele deve possuir bom poder de diluição e boa fluidez, além de apresentar uma boa distribuição do tamanho de partículas de modo a favorecer as condições de mistura (19). Embora os materiais de enchimento sejam normalmente inertes, eles podem afetar de maneira significativa as propriedades biofarmacêuticas, químicas e físicas dos comprimidos (20).

A lactose é um dos materiais de carga mais usados na produção de comprimidos. Entretanto, devido a baixa fluidez e compressibilidade, a lactose em sua forma regular não é utilizada na produção de comprimidos por compressão direta sem que ocorram algumas modificações nas suas características. Vários tipos de lactose estão disponíveis comercialmente, com diferentes propriedades físicas, tais como distribuição do tamanho de partículas e características de fluxo. Isso permite a seleção do material mais adequado para a aplicação desejada (21)

A lactose apresenta-se em duas formas isoméricas, a $\alpha$-lactose, que ocorre tanto na forma monoidratada como na forma anidra, e a $\beta$-lactose, que ocorre apenas na forma anidra. Para a compressão direta, frações cristalinas, grosseiras e peneiradas de $\alpha$-lactose monoidratada são utilizadas devido à sua melhor fluidez (8). A lactose anidra, composta de $70-80 \%$ de $\beta$-lactose anidra e $20-30 \%$ de $\alpha$-lactose anidra, é uma lactose sem água de hidratação. Comprimidos produzidos com lactose anidra geralmente apresentam rápida desintegração, boa friabilidade e baixa variação de peso, com ausência de fenômenos como aderência às partes da compressora e capping. Entretanto, não possui uma fluidez muito boa devido à presença de uma quantidade relativamente grande de finos $(7,5)$.

Dentre os diferentes tipos de lactose, a lactose spray-dried é o adjuvante mais recomendado para compressão direta, pois devido ao formato geralmente esférico de suas partículas, apresenta características de fluxo e aglutinação superiores às apresentadas pela lactose. A lactose spray-dried tem excelente fluidez dentre os materiais de carga e enchimento utilizados para compressão direta, entretanto possui problemas de compressibilidade. É efetiva quando constitui a porção majoritária do comprimido (mais de $80 \%$ ), porém não é efetiva em diluir fármacos de alta dose cuja natureza cristalina não seja compressível $(7,20,21,22)$. A lactose spray-dried, desenvolvida na década de 50, é produzida por diversos fornecedores e por esse motivo, as condições de processo utilizadas podem variar, influenciando tanto a fluidez como a compressibilidade. Dessa feita, uma maneira de evitar o surgimento desses problemas é a utilização de lactose oriunda de um mesmo fabricante. 
A utilização da celulose na produção de comprimidos teve início também na década de 50, com um produto denominado Solka-Floc ${ }^{\circledR}$. Todavia, por se tratar de um produto com pobres características de fluxo e de compressibilidade, deixou de ser usado. No início dos anos 60 , modificações no processo de obtenção da celulose originaram a celulose microcristalina, que até hoje permanece sendo o excipiente mais importante para a produção de comprimidos (7). Devido às suas propriedades, a celulose microcristalina possui múltiplas funções na fabricação de comprimidos. É considerado o melhor aglutinante seco para compressão direta, permitindo a obtenção de comprimidos resistentes à ruptura e à abrasão, sendo muito utilizada como material de enchimento. Apresenta também, propriedades desintegrantes apesar desse efeito não ser muito consistente e altamente dependente da formulação. Possui problemas como pobre capacidade de fluxo e sensibilidade à umidade, porém uma formulação adequada, que consiga garantir um bom escoamento do pó e bom preenchimento da matriz de compressão, pode contornar esses inconvenientes da celulose microcristalina (23).

Outra característica importante desse excipiente é apresentar baixa densidade bruta, alto potencial de diluição, baixo coeficiente de fricção e larga distribuição granulométrica, favorecendo o empacotamento. Pelo fato de ser altamente compressível, auto-lubrificante e desintegrante, tentativas tem sido feitas com o intuito de seu uso como único adjuvante nos comprimidos contendo fármacos de baixas doses. Contudo, foi observado que formulações contendo mais de $80 \%$ de celulose microcristalina podem diminuir a velocidade de dissolução de fármacos com baixa solubilidade aquosa (8).

Atualmente há diversos produtos contendo celulose microcristalina que são comercializados como excipientes para compressão direta, com destaque para Avicel $^{\circledR}$, Emcocel $^{\circledR}$ e Microcel $^{\mathbb{}}$. Tamanhos médio de partícula e conteúdo de umidade são as diferenças entre os diversos tipos de celulose micro${\text { cristalina } \text { Avicel }^{\circledR} \text { (Tabela 2) e Microcel }}^{\circledR}$ (Tabela 3). Os tipos de celulose microcristalina mais usadas são a 101 , que vem a ser o produto original, e a 102 , com um maior tamanho de partículas e que resulta em uma fluidez levemente melhor sem decréscimo na compressibilidade (7). Para sua utilização na produção de comprimidos por compressão direta, cada tipo de celulose microcristalina tem uma finalidade. A 101 e 102 são agentes de compressão de uso geral; a 103, 112 e 122 são utilizadas como agentes de compressão de baixa umidade, para substâncias ativas muito sensíveis a umidade; 200 e 250 , agentes de compressão de alta granulometria, utilizados para incrementar o fluxo e reduzir a variação de peso; 301 e 302, agentes de compressão de alta densidade, além de auxiliarem no fluxo; 500, agente de compressão e fluxo de alta granulometria, utilizado em fármacos de fluxo muito ruim (21).

Esses dois materiais, lactose spray-dried e celulose microcristalina, podem ser usados sozinhos em uma formulação de comprimidos por compressão direta como material de carga e enchimento com apropriados desintegrantes e lubrificantes, mas tem suas desvantagens. A celulose microcristalina, embora seja extremamente compressível e com propriedades desintegrantes, não apresenta fluidez adequada para produção de comprimidos com peso uniforme em processos de alta velocidade. A lactose, por outro lado, possui boa fluidez, mas é apenas moderadamente compressível e não tem propriedades desintegrantes. Entretanto, esses dois materiais quando usados em combinação fazem uma mistura quase ideal para formulações de comprimidos pela técnica de compressão direta (22).

Tabela 2: Celulose microcristalina - Avicel ${ }^{\circledR}$

\begin{tabular}{|c|c|c|}
\hline Tipo & $\begin{array}{c}\text { Tamanho médio } \\
\text { de particula }\end{array}$ & $\begin{array}{c}\text { Teor de } \\
\text { umidade }\end{array}$ \\
\hline PH 101 & $50 \mu \mathrm{m}$ & $5,0 \%$ \\
\hline PH 102 & $100 \mu \mathrm{m}$ & $5,0 \%$ \\
\hline PH 102SCG & $120 \mu \mathrm{m}$ & $5,0 \%$ \\
\hline PH 103 & $50 \mu \mathrm{m}$ & $3,0 \%$ \\
\hline PH 105 & $20 \mu \mathrm{m}$ & $5,0 \%$ \\
\hline PH 112 & $100 \mu \mathrm{m}$ & $1,50 \%$ \\
\hline PH 200 & $200 \mu \mathrm{m}$ & $5,0 \%$ \\
\hline
\end{tabular}

Outro material de enchimento bastante utilizado é o amido, porém em seu estado natural não possui as duas propriedades necessárias para a compressão: compressibilidade e fluidez. Quando o amido não é modificado, apresenta a tendência de não comprimir bem, além de incrementar o capping e a friabilidade. Várias tentativas de modificação foram realizadas com o intuito de melhorar suas características tecnológicas. $\mathrm{O}$ amido pré-gelatinizado é uma dessas modificações que, mesmo sendo primariamente um aglutinante para granulação úmida, pode ser modificado para torná-lo mais compressível e com boa fluidez. Quimicamente não difere muito do amido tradicional, e é comercializado com o nome de Starx $1500^{\circledR}(20)$

Por possuir mínimo potencial de diluição, o Starx $1500^{\circledR}$ geralmente não é utilizado como material de enchimento na compressão direta, e sim como desintegrante, sendo essa a sua principal vantagem, pois mantém as propriedades desintegrantes do amido e não influi em características da formulação como fluidez e compressibilidade. Mesmo sendo considerado um material de flu- 
xo livre, suas propriedades de fluxo são pobres quando comparadas com outros diluentes devido à grande superfície especifica do seu pó (7).

Sais inorgânicos de cálcio também são utilizados como materiais de enchimento. Dentre eles, o fosfato dicálcico diidratado $\left(\mathrm{CaHPO}_{4} \cdot 2 \mathrm{H}_{2} \mathrm{O}\right)$ é o mais comum, possuindo boa fluidez, não sendo necessária a adição de lubrificante, e é tão compressível como a celulose microcristalina e mais compressível que a lactose spray-dried. É disponível comercialmente com o nome de Emcompress ${ }^{\circledR}$.

Tabela 3: Celulose microcristalina - Microcel $^{\circledR}$

\begin{tabular}{|c|c|c|}
\hline Tipo & $\begin{array}{c}\text { Tamanho Médio } \\
\text { de Particula }\end{array}$ & $\begin{array}{c}\text { Teor de } \\
\text { Umidade }\end{array}$ \\
\hline MC 101 & $50 \mu \mathrm{m}$ & $<7,0 \%$ \\
\hline MC 102 & $100 \mu \mathrm{m}$ & $<7,0 \%$ \\
\hline MC 103 & $50 \mu \mathrm{m}$ & $<3,0 \%$ \\
\hline MC 122 & $100 \mu \mathrm{m}$ & $<3,0 \%$ \\
\hline MC 200 & $180 \mu \mathrm{m}$ & $<7,0 \%$ \\
\hline MC 250 & $230 \mu \mathrm{m}$ & $<7,0 \%$ \\
\hline MC 301 & $50 \mu \mathrm{m}$ & $<7,0 \%$ \\
\hline MC 302 & $100 \mu \mathrm{m}$ & $<7,0 \%$ \\
\hline MC 500 & $270 \mu \mathrm{m}$ & $<7,0 \%$ \\
\hline
\end{tabular}

Os fosfatos podem ser obtidosna forma pulverizada, com boa aplicação em via úmida e/ou compactação em rolos. Na sua forma granular tem aplicação em compressão direta, sendo a forma anidra a mais utilizada. Quando submetido a altas pressões na compressora, pode apresentar capping e laminação. Possui alta densidade, é muito abrasivo e não higroscópio, não se desintegra rapidamente e necessita de meio ácido para dissolução (7).

O fosfato dicálcico diidratado é relativamente insolúvel em água, porém comprimidos que contenham $50 \%$ ou mais dele se desintegram rapidamente. A utilização de fosfato dicálcico diidratado como material de enchimento pode acarretar em mudanças nas propriedades físicas dos comprimidos, tais como dureza, tempo de desintegração e tempo de dissolução do fármaco, além do seu uso não ser recomendável em altas concentrações em formulações contendo fármacos de baixa solubilidade em água (8).

Outro grupo de adjuvantes utilizados são os desintegrantes, que são adicionados à formulação a fim de promover e acelerar a desintegração do comprimido quando em contato com meios de natureza aquosa ou sucos digestivos. O objetivo principal é provocar a rápida desagregação do comprimido, bem como aumen- tar a área superficial dos fragmentos do mesmo a fim de liberar rapidamente o fármaco. A desintegração do comprimido está condicionada a solubilidade do fármaco, à força de compressão aplicada, à porosidade do comprimido e ao tipo e proporção de desintegrante adicionado à formulação $(9,10,23)$.

Os desintegrantes podem atuar por diferentes mecanismos: (a) por intumescimento quando em contato com líquidos aquosos, favorecendo a separação das partículas do comprimido, o que causa um aumento na superfície específica, provocando um aumento na velocidade de dissolução; (b) dissolução em água, originando canais capilares no comprimido, o que facilita a entrada de fluídos e desintegra o comprimido; (c) reação com água, no caso dos comprimidos efervescentes, liberando um gás, geralmente o $\mathrm{CO}_{2}(9,10,24)$.

Também podem ser encontradas variedades de desagregantes denominados superdesintegrantes. Eles são assim denominados por possuírem grande poder de desintegração com a utilização de pequenas quantidades (entre 0,5 e $5 \%$ ), entretanto sua eficiência depende do método de fabricação e/ou características físico-químicas da formulação (25). Dentre esses superdesintegrantes destacam-se a croscarmelose sódica e o amidoglicolato de sódio.

A croscarmelose sódica, um derivado sódico da celulose, apresenta intumescimento quando em contato com água, podendo aumentar seu volume inicial de quatro a oito vezes, gerando um grande poder de desintegração. Possui efeitos positivos na lipodisponibilidade de alguns fármacos, além de exercer pouca influência nas características tecnológicas dos comprimidos, como dureza e friabilidade $(21,26)$. $\mathrm{O}$ uso da croscarmelose sódica pode também influir no poder de intumescimento de outros adjuvantes, como a celulose microcristalina (27). A concentração de uso da croscarmelose sódica como desintegrante de comprimidos varia de 0,5 à 5,0 $\%(21)$.

O amidoglicolato de sódio é um derivado substituído do amido, sendo sua atividade desintegrante afetada pelo grau de ligações cruzadas e da extensão da carboximetilação. Possui uma alta capacidade de intumescimento, podendo aumentar seu volume em até trezentas vezes, sendo dessa forma muito ativa em pequenas concentrações. A presença de excipientes hidrofóbicos, como os lubrificantes, não afeta a sua eficiência como desintegrante. Por outro lado, um aumento na pressão de compressão também afetar o tempo de desintegração do comprimido contendo amidoglicolato de sódio $(16,20)$. A concentração usual empregada em formulações é entre $2 \%$ e $8 \%$, com uma concentração ótima de $4 \%$ (21)

No processo de compressão pode ocorrer atrito e/ ou fricção entre os participantes da compressão e, para 
contornar tais problemas lança-se mão de agentes lubrificantes que tendem a equalizar a distribuição da pressão em um comprimido (20) Os lubrificantes podem ser classificados de acordo com a sua função desempenhada em uma formulação: (a) deslizantes (diminuem a fricção interparticular, melhorando o fluxo); (b) antiaderentes (diminuem a aderência dos comprimidos aos punções e à matriz); (c) lubrificantes propriamente ditos (reduzem a fricção entre as partículas durante a compressão, assegurando uma melhor transmissão da força de compressão através do pó). Essas três atividades podem ser verificadas de forma simultânea em um mesmo lubrificante, porém a eficiência de tais funções será de menor intensidade $(14,15)$.

O estearato de magnésio é o lubrificante mais comumente utilizado na fabricação de comprimidos, em parte devido a sua tendência à migração, durante a compressão, até a interface com a parede da matriz, alcançando altas concentrações na superfície do comprimido (15). Parâmetros como estrutura dos cristais, tamanho de partículas e área de superfície específica, afetam a eficiência do estearato de magnésio (28). Pelo fato de ser hidrofóbico, o estearato de magnésio pode retardar a dissolução de um fármaco em uma forma farmacêutica sólida. Assim, a menor concentração possível desse lubrificante deve ser utilizada nas formulações. Geralmente a concentração usual de estearato de magnésio encontra-se entre $0,25 \%$ e $0,5 \%$ (21)

A velocidade de dissolução e a friabilidade de comprimidos são afetadas de forma antagônica pela utilização do estearato de magnésio. Mesmo ambas diminuindo à medida que se aumenta o tempo de mistura de um pó que contenha estearato de magnésio, a redução de friabilidade é desejada para garantir a integridade física do comprimido formado; por outro lado, a redução da velocidade de dissolução pode afetar a liberação do fármaco, diminuindo-a. Por isso o tempo de mistura do material em pó com o estearato de magnésio deve ser controlado (21).

Outro adjuvante utilizado como lubrificante é o dióxido de silício coloidal. Utilizado como mediador de fluxo, pois o pequeno tamanho das partículas e a grande área de superfície específica acarretam características de fluxo desejado, que podem ser exploradas para melhorar as propriedades de fluxo de pós. Além disso, é efetivo em pequenas concentrações. Evita a aderência do material à matriz e aos punções, atuando por interposição entre as partículas formando uma camada protetora que diminui a fricção interparticular e a tendência à coesão, além de favorecer o enchimento homogêneo da matriz, consegue se introduzir entre as irregularidades dos grânulos. O dióxido de silício coloidal é um pó higroscópico, mas absorve grandes quantidades de água sem se liquefazer e, da mesma forma que o estearato de magnésio, apresenta uma concentração usual de $0,25 \%$ à 0,5 $\%(9,21,22)$.

Com o intuito de aprimorar a produção de comprimidos por compressão direta, a indústria farmacêutica passou a desenvolver novos excipientes que combinam as funções e/ou vantagens de diferentes insumos em um único produto. Esses novos insumos são denominados excipientes co-processados, pois são resultado da combinação de dois ou mais excipientes misturados em proporções adequadas, que após um processamento farmacotécnico geram um novo produto com melhores propriedades para compressão direta que sua simples mistura física (29). O principal objetivo de um co-processado é obter um produto que agregue valor na relação preço/ funcionalidade. (30)

A produção desses co-processados baseia-se na alteração física dos produtos envolvidos sem a necessidade de alteração da estrutura química dos mesmos. $\mathrm{O}$ desenvolvimento de um excipiente co-processado diretamente compressível inicia com a seleção dos excipientes a serem combinados, sua proporção, seleção do método de preparação para otimizar o produto relativo ao seus parâmetros físico-químicos desejados e termina com o intuito de evitar ao máximo a variação lote-a-lote. Contudo, a principal limitação em uma mistura de excipientes co-processados é que a razão dos excipientes utilizados nem sempre são as mesmas para os diferentes insumos ativos utilizados na produção de comprimidos. Pelo fato de não apresentarem aceitação oficial de farmacopéias, uma combinação de excipientes muitas vezes só é aceita na indústria farmacêutica a partir do momento em que esse apresente uma vantagem bastante significativa para a produção de comprimidos quando comparado com a mistura simples dos mesmos excipientes (30). A Tabela 4 apresenta alguns exemplos de excipientes co-processados, com seu nome comercial, suas composições e as características do mesmo $(29,30)$.

\section{CONSIDERAÇÕES FINAIS}

O emprego de adjuvantes multifuncionais tem se mostrado uma alternativa relevante no desenvolvimento de comprimidos por compressão direta. Isto se deve à possibilidade de combinação de diversas propriedades em um único adjuvante. Por esse motivo, a seleção adequada dos adjuvantes farmacêuticos a serem empregados é de fundamental importância no planejamento de formulações farmacêuticas $(29,30)$. Esse desenvolvimento deve ser feito de maneira científica, por meio de estudos pré-formulação, o que facilita a escolha dos adjuvantes tecnológicos mais viáveis na elaboração de um produto farmacêutico. 
Tabela 4: Exemplos de excipientes co-processados.

\begin{tabular}{|c|c|c|}
\hline Nome comercial & Composição & Características \\
\hline Cellactose $^{\circ}$ & $\begin{array}{l}75 \% \text { celulose em pó }+25 \% \text {-lactose- } \\
\text { monohidratada }\end{array}$ & $\begin{array}{l}\text { Excelente compressibilidade e compactabilidade; } \\
\text { boa desintegração }\end{array}$ \\
\hline MicroceLac $^{\circ}$ & $\begin{array}{c}75 \% \alpha \text {-lactose-monohidratada }+25 \% \text { celulose } \\
\text { microcristalina }\end{array}$ & Excelente compressibilidade e compactabilidade \\
\hline Starlac ${ }^{\circ}$ & $\begin{array}{l}75 \% \alpha \text {-lactose-monohidratada }+25 \% \text { celulose } \\
\text { microcristalina }\end{array}$ & $\begin{array}{l}\text { Excelente compressibilidade e desintegração; boa } \\
\text { compactabilidade }\end{array}$ \\
\hline Ludipress $^{\circ}$ & $\begin{array}{c}934,4 \% \alpha \text {-lactose-monohidratada }+3,2 \% \text { PVP } \\
+3,4 \% \text { Crospovidona }\end{array}$ & Excelentes propriedades de fluxo \\
\hline Pharmatose $^{\circ}$ & $95 \% \beta$-lactose $+5 \%$ lactitol anidra & $\begin{array}{l}\text { Boa fluidez; boas propriedades ligantes; baixa } \\
\text { retenção de água em alta umidade }\end{array}$ \\
\hline
\end{tabular}

\section{REFERÊNCIAS}

1. Rudnic E; Schwartz J. Oral Solid Dosage Forms In Gennaro, A. Remington: The science and practice of pharmacy. $20^{\text {th }}$ ed Philadelphia: Lippincott Williams \& Wilkins, 2000.

2. Armstrong N. Tablet Manufacture by Direct Compression In: Swarbrick J. Encyclopedia of Pharmaceutical Technology. $3^{\text {rd }}$ ed. New York: Informa Healthcare, v.1, pp. 3673-83, 2007

3. McCornick D. Evolutions in direct compression. Pharmaceutical Technol 2005. April:52-62

4. Hoag S; Dave V; Moolchandani, V. Compression and Compaction. In:. Augsburger S; Hoag S. Pharmaceutical Dosage Forms: Unit Operations an d Mechanical Properties (L). $3^{\text {rd }}$ ed.: Vol 1 New York: Informa Healthcare, pp. 55-619, 2008.Alderbon G. Tablets and Compaction. In Aulton M. Pharmaceutics. The Science of Dosage Form Design. $2^{\text {nd }}$ ed. Edinburgh: Churchill - Livingstone, 2002.

5. Jivraj M; Luigi M; Thomson C. An overview of the different excipients useful for the direct compression of tablets. Pharm. Sci \& Tech Today, 2000. 2(3):58- 63

6. Shangraw R. Compressed Tablets by direct compression In Lieberman H.; Lachman L; Schwartz J. Pharmaceutical Dosage Forms: Tablets. $2^{\text {nd }}$ ed, New York: Marcel Dekker Inc, 1989, v.1.

7. Bolhuis $\mathrm{G} \&$ Chowhan Z. Materials for direct compression. In Alderbon G; Nystrom C. Pharmaceutical Powder Compaction Technology. New York: Marcel Dekker, 1996.

8. Hausner HH. Friction conditions in a mass of metal powder. Int. J. Powder Metall. 1967. 3:7-13

9. Carr RL. Evaluating flow properties of solids. Chem. Eng. 1965.72(2):163-168.

10. Guoyt JC; Arnaud P; Becourt P; Cassiere JP; Chulia D; Duchêne D; Ferrand D; Gouaihardou J; Langlois C; Lequien C; Mafroid F; Pourcelot Y; Renoux R; Segot S; Toure P. Commentaires relatifs aux methods générales d'analyse des formes orales solides récemment introduites dans les pharmacopeés française et européenne. Rapport d'une commission. SFSTP S.T.P. 1995. Pharma Pratiques 5(6):482-494

11. Ehrhardt L.; Schindler E. Pharmazeutische Granulate. 1. Mitteilung. Pharmazeutische Industrie. 1980. 41(12):96-103

12. Thomas C; Pourcelot Y. Preformulation of five commercial celluloses in drug development rheological and mechanical behavior. Drug Dev. Ind. Pharm 1991. 19(15):1947-1964

13. Voigt R. Pharmazeutische Technologie für Studium und Beruf. 9. vollst. Überarb. Aufl. Stuttgart: Deutsche Apotheker, 2000.

14. Vila Jato J. Tecnología Farmacéutica. Madrid: Síntesis, 2001, 2. v.

15. Soares LAL; Petrovick PR. Física da compressão. Cad Farmácia 1999. 15(2):65-9
16. Çelik M. The Past, Present, and Future of Tableting Technology. Drug Dev. Ind. Pharm. 1996. 2(1):1- 10

17. Moretto LD. Fatores que influem na biodisponibilidade de fármacos e medicamentos. Pharmaceutical Technol. 1999. 46 - 48

18. Augsburger L; Zellhofer M. Tablet Formulation. In Swarbrick J. Encyclopedia of Pharmaceutical Technology $3^{\text {rd }}$ ed. New York: Informa Healthcare, 1:3641 - 52, 2007.

19. Banker GS; Peck GE; Baley G. Tablet formulation and design. In Lieberman H; Lachman L. Pharmaceutical Dosage Forms: Tablets. New York: Marcel Dekker, 1980. v.1.

20. Kibbe A. Handbook of Pharmaceutical Excipients. $6^{\text {th }}$ ed. Washington, DC: American Pharmaceutical Association, 2009.

21. Lerk C. Consolidation and compaction of lactose. Drug Dev. Ind. Pharm 1993 19(17/18):2359-3998

22. Doelker E. Comparative compaction properties of various microcrystalline cellulose types and generic products. Drug Dev. Ind. Pharm. 1993. 19(17/18): 2119-2141

23. Le Hir A. Farmacia Galénica. Barcelona: Masson, 1995.

24. Ferrero C; Muñoz N; Velasco MV; Muñoz-Ruiz A; JuménezCastellanos R. Disintegrating efficiency of croscarmellose sodium in a direct compression formulation. Int. J. Pharm. 1997. 147(1):11-2

25. Amidon G; Secreast P; Mudie D. Particle, Powder and Compact Characterization. In Qiu Y; Chen Y; Zhang G. Developing Solid Oral Dosage Forms: Pharmaceutical Theory and Practice) New York: Elsevier, pp. 163- 86, 2009.

26. Lima Neto A. Determinação das características de intumescimento de adjuvantes. 1996. Dissertação (Mestrado em Ciências Farmacêuticas) - Universidade Federal do Rio Grande do Sul. Porto Alegre.

27. Danish FQ; Parrot EL. Effect of concentration and size of lubricants on flow rate of granules. J. Pharm. Sci. 1971. 60(5):752 - 752

28. Barreto L; Cunha-Filho M. Excipientes Co-processados para Compressão Direta de Comprimidos. Lat. Am. J. Pharm. 2009. 28(2): 304-12

29. Gohel M; Jogani P. A review of co-processed directly compressible excipients. J Pharm Pharmaceut Sci 2005. 8(1):76-93

30. Stamm A. Process and dosage form controls: formulation factors. 1989. Drug Dev. Ind. Pharm. 15(6/7): 965 - 974

31. Staniforth J. The design and use of tableting excipients. Drug Dev. Ind. Pharm 1993. 19(17/18):2273 - 2308 